\title{
Tactile representation in somatosensory thalamus (VPL) and cortex (S1) of awake primate and the plasticity induced by VPL neuroprosthetic stimulation
}

\author{
Weiguo Song ${ }^{1}$, Mulugeta Semework ${ }^{2}$
}

1. Dept. of Physiology and Pharmacology, SUNY Downstate Medical Center. NY 11203.

2. Joint Graduate Program in Biomedical Engineering SUNY Downstate and NYU-POLY. NY 11203. Current address: Department of Neuroscience, Columbia University, 1051 Riverside Drive, Unit 87, New York, N.Y. 10032

Corresponding author:

Weiguo Song, Department of Physiology, Pharmacology and Neuroscience, City College of the City University of New York, New York, NY,10031, USA

weiguo.song@yahoo.com

Keywords: somatosensory cortex, thalamus, plasticity, VPL stimulation, tactile encoding, mutual-information 


\section{Abstract}

To further understand how tactile information is carried in somatosensory cortex (S1) and the

thalamus (VPL), and how neuronal plasticity after neuroprosthetic stimulation affects sensory encoding, we chronically implanted microelectrode arrays across hand areas in both S1 and VPL, where neuronal activities were simultaneously recorded during tactile stimulation on the finger pad of awake monkeys. Tactile information encoded in the firing rate of individual units (rate coding) or in the synchrony of unit pairs (synchrony coding) was quantitatively assessed within the information theoretic-framework. We found that tactile information encoded in VPL was higher than that encoded in $\mathrm{S} 1$ for both rate coding and synchrony coding; rate coding carried greater information than synchrony coding for the same recording area. With the aim for neuroprosthetic stimulation, plasticity of the circuit was tested after 30 minutes of VPL electrical stimulation, where stimuli were delivered either randomly or contingent on the spiking of an S1 unit. We showed that neural encoding in VPL was more stable than in S1, which depends not only on the thalamic input but also on recurrent feedback. The percent change of mutualinformation after stimulation was increased with closed-loop stimulation, but decreased with random stimulation. The underlying mechanisms during closed-loop stimulation might be spiketiming-dependent plasticity, while frequency-dependent synaptic plasticity might play a role in random stimulation. Our results suggest that VPL could be a promising target region for somatosensory stimulation with closed-loop brain-machine-interface applications.

\section{Introduction}

To feel a sense of touch, tactile information needs to be transmitted from peripheral receptors through the spinal cord, dorsal column nuclei, and ventral posterior lateral nucleus (VPL) of the thalamus on to the somatosensory cortex. Somatosensory prosthetics has shown promising results to replace lost function (Joseph E. O'Doherty, 2011) due to damage to the structures in this pathway. However, whether and how such an intervention affects normal sensory 
processing is not clear. Moreover, only limited studies have been conducted on simultaneous recordings in S1 and VPL in awake monkeys (Vazquez et al., 2013), mainly due technical difficulties. In this paper, we will address the following two key questions.

The first question is how tactile information is represented in $\mathrm{S} 1$ and VPL, and how it is transmitted between them. In peripheral organs, stimulus is generally encoded in the firing rate of sensory receptors (Adrian, 1926). Rate coding has also been found in both VPL and S1 (Vazquez et al., 2013), and tactile information in S1 is thought to be relayed through VPL of the thalamus (Constantinople and Bruno, 2013; Vazquez et al., 2013). Anatomically, cortical neurons only receive limited information from the thalamus (da Costa and Martin, 2009). Thus, how sensory information is represented or transmitted in the thalamocortical loop needs further study. As sensory information needs to be processed efficiently in dynamic environments at both S1 and VPL (Bernander et al., 1994; Douglas et al., 1995; Montemurro et al., 2007), other non-rate encoding strategies might exist (Gautrais and Thorpe, 1998; Nicolelis et al., 1998; Salinas and Sejnowski, 2001). Synchronization, as a coincidence detector, is a fast encoding/decoding method and has been observed in S1 not only during demanding or cogitative tasks (Steinmetz et al., 2000; Panzeri et al., 2001; Alloway et al., 2002; Niebur et al., 2002; Roy et al., 2007), but also under quiet awake (Song and Francis, 2013) or anesthetized conditions (Reed et al., 2008). This temporal coding was also found in the thalamus of rats (Montemurro et al., 2007), cats (Reinagel and Reid, 2000) and monkeys (Jones, 2001). Thalamic synchrony could help to drive cortical activation (Bruno and Sakmann, 2006), and synchrony might participate in cortical and thalamic sensory processing. Combining simultaneous neuronal recordings in VPL and S1 with information theory based analyses, we quantified tactile information encoded in both areas (with either rate coding of individual units or synchrony coding of unit pairs). We also investigated the direction of information flow between these structures. 
The second question we will address concerns VPL and S1 plasticity after electrical stimulation in VPL. With the specific aim of designing a robust somatosensory prosthetic, which ideally does not change the intrinsic sensory circuit, we tested the plasticity of S1 and VPL after different stimulation protocols. Plasticity is commonly observed in S1 during development (Feldman et al., 1999; Vue et al., 2013), learning (Nudo et al., 1996; Ganguly and Carmena, 2009), after injury (Kaas et al., 1983), and after stimulation (Keller et al., 1992a; Jackson et al., 2006; Rebesco et al., 2010). VPL, the traditional somatosensory relay center, has been thought to stop reorganizing in adulthood (Chun et al., 2013). However, functional reorganization has been found after injury (Jones and Pons, 1998; Weng et al., 2000). Recent evidence demonstrates that the somatosensory map in S1 depends on the plasticity of thalamocortical axons (Datwani et al., 2002; Vue et al., 2013), and that thalamocortical input could be plastic throughout lifetime (Oberlaender et al., 2012). For instance, cortical synaptic proliferation was observed after four days of VPL stimulation (Keller et al., 1992a). Thus, where plasticity is initiated is still controversial (Kaas, 1999; Fox et al., 2000). Additionally, there are no adequate data showing how it affects sensory encoding and whether or not it is suitable for somatosensory prosthetic application. To this aim, we tested the plasticity of the underlying circuit by utilizing information-theoretic criteria after either random or closed-loop electrical stimulation in the VPL.

\section{Results}

\subsection{Tactile information encoding in S1 and VPL}

A total of 22 sessions (monkey G: 12 and monkey A: 10) were recorded during tactile stimulation, from which 313 units (monkey G: 84 and monkey A: 229) were in VPL and 1545 units (monkey G: 932 and monkey A: 613) were in S1. As previously reported (Reed et al., 2011), we found neuronal activities in both S1 and VPL which manifested as quick responses to tactile stimulation on the finger pad (see two example units in figure $2 \mathrm{~A}$ and $\mathrm{C}$ ). Synchrony, the simultaneous firing of two units after binning the spike trains at $1 \mathrm{~ms}$ resolution, also modulated 
with tactile stimulation (Fig 2 B and D). Synchrony variation was not simply a result of firing rate modulation, as synchrony was over the $95 \%$ confidence interval of the trial shuffled ones, where the responses of two units were randomly chosen from different trials during tactile stimulation. This example also shows that even if the rate modulation was similar in both VPL and S1, synchrony could be different (Fig 2 B and D).

To quantify tactile encoding in neuronal activities, we applied information theory measures to directly quantify the information encoded in the firing rate of individual unit (rate coding) and in the synchrony of unit pairs (synchrony coding). The number of units that significantly encoded tactile information with rate coding was significantly higher in VPL than in $S 1(p<0.01$, for kstest2 and ttest2) (Fig 3 A and B). Similar to rate coding, tactile information with synchrony coding of unit pairs was also significantly greater in VPL than in $S 1(p<0.01$, for kstest2). Not only was the encoded information in VPL significantly higher under either coding strategy (rate coding or synchrony coding), higher percentage of units encoded tactile information as well (Fig $3 \mathrm{C}$ and $\mathrm{D}$ ). To make sure that our analysis was not affected by sampling issues during probability calculation, we compared the information based analyses with linear modulation in amplitude of either firing rate or synchrony rate. This was done by regression analysis (firing rate or synchrony rate regressed on the mutual-information from rate coding or synchrony coding). The mutual-information from rate coding agrees more with modulation of firing rate in VPL $(R$-squared $=0.58, p<0.05)$ than in $S 1(R$-squared $=0.22, p<0.05)$. Synchrony coding was only significantly correlated with the amplitude of synchrony rate in VPL (R-squared $=0.50$, $p<0.01$ ), not in $S 1$ (R-squared $=0.00, p>0.05)$. In both VPL and $S 1$, mutual-information from rate coding was significantly higher than that from synchrony coding $(p<0.01$, for kstests and ttest2). Tactile information encoded in VPL was significantly higher than that in $\mathrm{S} 1$, for both rate and synchrony coding ( $p<0.01$, for kstests and ttest 2$)$.

\subsection{Effect of VPL neuroprosthetic stimulation on thalamocortical circuits}


To investigate plasticities within thalamocortical circuits after neuroprosthetic usage, we studied neuronal response changes to tactile stimulation after electrically stimulating the VPL either based on a closed-loop (spike-triggered) or a random microstimulation patterns (see methods). The changes of sensory encoding were quantified from information criteria. A total of 8 sessions in one monkey $(A)$ were recorded, from which 176 units in VPL and 512 units in S1 were analyzed. In VPL, the effect of random stimulation was not significantly different from that of closed-loop stimulation for both rate and synchrony coding ( $p<0.01$ for kstest2) (Fig 4 A and B). On the other hand, in $S 1$, the two effects were significantly different $(p<0.01$ for kstest2); in either rate (Fig 4 C) or synchrony coding (Fig 4 D). Random stimulation only significantly decreased mutual-information from synchrony coding $(p<0.01$ for ttest2), not that from rate coding. No significant difference was found after closed-loop stimulation with either rate coding or synchrony coding. We also looked at the percentage of units that significantly encoded tactile information (trial shuffling: $n=20$ ). For rate coding in VPL, although the distribution of the information values was not significantly different, the number of units that significantly encoded tactile information decreased by around $7 \%$ after random stimulation, compared to the $5 \%$ increase after closed-loop stimulation (Fig 4 A). A similar pattern was found in S1: a $3 \%$ increase after closed-loop and a $3 \%$ decrease after random stimulation (Fig 4 C). Synchrony coding in VPL showed less than $2 \%$ change after either closed-loop stimulation or random stimulation, and there were no significant changes in information value (Fig 4 D).

Apart from improving encoding efficiency (mutual-information value) or recruiting more units individually, plasticity could also manifest as change in correlation between the spiking of unit pairs. Following our previously used method (Song and Giszter, 2011), we first calculated the traditional firing rate correlation coefficient between VPL and S1 unit pairs. The conditioning unit pairs (between triggering S1 units and stimulating VPL units) showed an increased correlation after closed-loop stimulation. The correlation coefficient was greater between conditioning pairs 
than between non-conditioning pairs after closed-loop stimulation (Fig 5). By using a transfer entropy (TE) approach that provides directional information for the information transmission, we further looked at the coupling between VPL and S1 from (entire unit pairs). TE Figure 6 A and B show directional coupling to be from VPL to $S 1$ than in the reverse direction. Although the direction of information flow did not significantly change (kstest2, p>0.05) after either closedloop or random stimulation, closed-loop stimulation showed significantly larger changes in information transfer from VPL to S1 than random stimulation did (Fig $6 \mathrm{D}$ ). This agrees with the correlational analysis, where closed-loop stimulation increased correlation while random stimulation deceased it (Fig 5).

As VPL is thought to be an information regulation center between dorsal column nuclei and the cortex, thalamocortical circuits could be modeled as electrical amplifiers, where VPL responses act as an input and S1 responses as output and a gain $(\mathrm{K})$ representing an intrinsic feature of the circuit. If stimulation did not alter the circuit pathway, then the output (tactile ensemble response of S1) and input (tactile ensemble response of VPL) will change proportionally. Accordingly, we regressed the gain after stimulation (Kpost) over the gain before stimulation (Kpre). The correlation between the gains was significant for both closed-loop (Fig 7 A: R$\mathrm{sq}=0.87, \mathrm{p}<0.05$ for regression) and random stimulation (Fig $7 \mathrm{~B}$ : R-sq=0.97, $p<0.05$ for regression). Both closed and open loop stimulation could change VPL and S1 responses. Interestingly, the thalamocortical circuit was linear and stable only after closed-loop stimulation. These facts were demonstrated by a unit slope and zero crossing of the regression line. This suggests that closed-loop stimulation did not change the underlying circuit and thus the plasticity of the circuit might depend on the stimulation protocols used.

\section{Discussion}

\subsection{Tactile information encoding in VPL and S1}

Sensory information in S1 is thought to be relayed through VPL (Jones, 1991), but there is only a limited number of synaptic projections to S1 (da Costa and Martin, 2009). Based on 
information analyses (Shannon, 1948), we quantified tactile information encoded in neuronal activities with rate coding and synchrony coding (Borst and Theunissen, 1999). Similar to what has been found (Camarillo et al., 2012), our results indicate that VPL appears to be a reliable tactile encoding center. With a spike train classifier during a sensory detection task, it was found that tactile detection accuracy of single neurons is similar in VPL and S1, but VPL relies more heavily on fast rate modulations than S1 does (Vazquez et al., 2013). We found that VPL encoded significantly greater information and recruited more tactile encoding units than S1 did. This might come from our recording units in both S1 and VPL showing fast responses to tactile stimulation. Compared with the active sensory detection task (Vazquez et al., 2013), where S1 was strongly involved in learning or cognition, our non-behavioral resting task might represent intrinsic features of the neuronal encoding. However, how such representations give rise to perception, memory, and decision needs further research (Parker and Newsome, 1998; Romo and de Lafuente, 2013). The lesser mutual-information in S1 (compared to that in VPL) could also arise from the limited number of synaptic projections from VPL to S1. Our data also indicate that rate coding might be a general encoding strategy used across different areas, while tactile information could also be encoded in synchrony. Synchronization is mostly observed during high-level demanding tasks or in dynamic environments in S1 (Nicolelis et al., 1998; Steinmetz et al., 2000; Panzeri et al., 2001; Salinas and Sejnowski, 2001; Niebur et al., 2002; Roy et al., 2007; Reed et al., 2008; Song and Francis, 2013). The thalamus also shows temporal coding (Reinagel and Reid, 2000; Montemurro et al., 2007; Stanley et al., 2012) and might be involved in high-level tasks as well (Komura et al., 2001; Vazquez et al., 2012). We do not know whether or not rate and synchrony coding are independent of each other (Huxter et al., 2003), but synchrony coding in our data was not a simple byproduct of the rate coding, as some units encoded only in synchrony but not in firing rate, and a smaller number of units showed tactile encoding in desynchronization (data not shown). Synchrony in the thalamus was found very effective in driving cortical targets (Bruno and Sakmann, 2006; Stanley et al., 2012), and 
could control information flow to the cortex (Wang et al., 2010). Thus, tactile information encoded in S1 could be increased by either recruiting additional inputs from the thalamus, or by increasing the encoding efficiency. Our observation that synchrony coding is stable (Fig 4 B) also suggests that it is an important regulator in VPL, and might contribute to stabilizing information transmission to $\mathrm{S} 1$ under dynamic conditions.

It was reported that the thalamus could modulate the cortex directly (Viaene et al., 2011). The maximum TE presented with a long-binning window (100ms) (Fig $5 \mathrm{C}$ ) also suggests that tactile information transmission between the thalamus and the cortex was not a simple synaptic effect. Moreover, recurrent networks might exist within the cortex, thalamus or between them. Compared with the limited projections from the thalamus to the cortex, corticothalamic projections are up to 10-fold greater in number (Liu et al., 1995), and cortical information could modulate the sensory processing in the thalamus (Shin and Chapin, 1990; Li and Ebner, 2007). Our S1 arrays mostly sampled neurons in deep layers of area 1 (layer IV to layer VI), where the major projections from VPL to $\mathrm{S} 1$ as well as the feedback between them are located. Units in these layers also have an active role in integration of thalamocortical inputs from local and distal intracortical excitatory inputs (Douglas et al., 1995) and are even directly activated by the thalamus (Constantinople and Bruno, 2013). In agreement with early reports (Castro-Alamancos, 2002), we also found that information flow increased during tactile stimulation compared with resting states rates.

\subsection{Plasticity of the thalamcortical circuit}

Plasticity could be arising from different rules, such as spike-timing-dependent plasticity (STDP), and activity-dependent as well as frequency-dependent synaptic plasticity (Kaas, 1991; Buonomano and Merzenich, 1998; Rioult-Pedotti et al., 2007; Feldman, 2009). Applying two neuroprosthetic stimulation paradigms (random stimulation vs. closed-loop stimulation in VPL) (Keller et al., 1992b; Rebesco et al., 2010; Song et al., 2013), we quantified the plasticity in VPL 
and $\mathrm{S} 1$ in terms of changes in mutual-information and the direction of information flow using information criteria measures (Shannon, 1948). Although synaptic proliferations were observed after four days of persistent VPL stimulation (Keller et al., 1992b), we did not find significant changes (looking at the entire population) in either rate or synchrony coding after closed-loop stimulation in VPL. Synchrony-coded information significantly decreased only after random stimulation. There were significant increases in the correlations between the conditioning unit pairs after closed-loop stimulation (Fig 5). This might indicate that although 30 minutes of stimulation in our paradigm was not long enough to induce anatomical changes, the two stimulation protocols caused clear distinctions in VPL and S1. In line with previous observations that training-induced cortical plasticity originates only in S1 and not VPL (Diamond et al., 1994; Wang et al., 1995), our results also show that plasticity is strong in S1 and depends on the protocol used (Fig 4). For both rate coding and synchrony coding, only S1 showed significant differences after closed-loop stimulation (compared to random stimulation) (Fig $4 \mathrm{C}$ and D). The small change in the number of encoding units also suggests that $\mathrm{S} 1$ plasticity induced by VPL stimulation is most likely caused through increased encoding efficiency rather than through recruitment of more units (Fig $4 \mathrm{C}$ and D). VPL plasticity might be regulated through the number of tactile encoding units. In agreement with a top-down modulation of tactile input (Shin and Chapin, 1990; Ghosh et al., 1994; Pais-Vieira et al., 2013), our results provide additional evidence that networks might be co-modulated, as manifested by a unit transmission gain during our closed-loop paradigm (Fig 7A). Cortically-induced changes in the thalamus depend on its and cortical neurons sharing the same response properties (Li and Ebner, 2007). Thus, cortical plasticity induced during closed-loop VPL stimulation might share a similar receptive field as well, as the triggering units in $\mathrm{S} 1$ were chosen from tactile-responsive cells in our closed-loop paradigm. How functionally-different cortical units affect thalamic information processing needs further research. On the other hand, random stimulation might behave as an interrupter. The information input from random VPL stimulation did not show any functional 
similarity with cortical units, which decreases both the transmission gain and encoding efficiency in S1. Further, the thalamus and cortex might interact with each other. It was found that S1 plasticity could be through thalamocortical synapses (Blundon et al., 2011). Our significant direction of information flow shift (Fig 6 D) might be the result of this plasticity. During closedloop stimulation, VPL stimulation-induced firing of VPL neurons was contingent on the firing of pre-synaptic S1 neurons (5 ms delay), which was within the STDP window, and thus the plasticity might be based on STDP. The firing of VPL neurons caused by random stimulation depended only on stimulation frequency, not timing. It is thus possible that spike-timingdependent plasticities may be the underlying mechanisms during closed-loop stimulation, while frequency-dependent synaptic plasticity might play a role in random stimulation (Ito and Schuman, 2007; Nikolaev et al., 2013).

\subsection{Closed-loop somatosensory neuroprosthetics implications}

How to provide somatosensory feedback to brain-machine-interfaces is a hot topic recently (Suminski et al., 2010; Heming et al., 2011; O'Doherty et al., 2011), but where to target for stimulation, and whether it is stable after persistent stimulation, are still open questions. Currently, somatosensory feedback is attempted dominantly through electrical stimulation in the somatosensory cortex (O'Doherty et al., 2011). On the other hand, the thalamus, which is the main sensory gateway to the cortex, could be an alternative target region for this application (Heming et al., 2011). Our findings that there is greater tactile information encoding and smaller plasticity after electrical stimulation in VPL than in S1 support this view. Depending on the desired goals shaping the underlying circuit, either closed-loop or random stimulation protocols can be applied.

\subsection{Conclusion}

We found that units in both VPL and S1 encode tactile information. Rate coding is more reliable than synchrony coding. Tactile encoding in VPL is more reliable than tactile encoding in S1. 
Synchrony coding could also play a role in stabilizing tactile information transmission between VPL and S1. Compared with the cortex, VPL is less plastic. The plasticity in S1 depends not only on thalamocortical inputs, but also on its own recurrent feedback. The underlying mechanism of closed-loop stimulation might be STDP (Bi and Poo, 1998; Song et al., 2000; Dan and Poo, 2004). Frequency-dependent plasticity might be dominant for random stimulation (Ganguly and Poo, 2013). Our results suggest that VPL could be another target region to provide sensory feedback. Closed-loop stimulation in VPL could further improve the sensory transmission efficiency without changing the intrinsic features of the thalamocortical circuit.

\section{Materials and Methods}

Two monkeys (G: male bonnet, $6.5 \mathrm{Kg}$; $\mathrm{A}$ : male rhesus, $5.3 \mathrm{Kg}$ ) were used in these experiments. Care and treatment of the animals during all stages of the experiments conformed to the procedures approved by the Division of Laboratory Animal Resources and Institutional Animal Care and Use Committee (IACUC: protocol \# 10-487-10) of SUNY Downstate Medical Center. All efforts were made to minimize distress. Head restrain was started at least 6 weeks after headpost implantation. Head restraints were adjusted to the subjects' comfort, and connection of headstages with the array connectors did not cause any stress to the animal.

\subsection{MRI Imaging and Surgical Procedure}

The implantation of VPL electrode was guided by MRI imaging. Under Ketamine (20 mg/kg) anesthesia, brain structural images were acquired (3T MRI, Siemens Com.) with the monkeys heads mounted in a stereotaxic frame in a sphinx position. In order to get accurate anatomical location of the brain structures, vitamin E fiducial markers were affixed to the frame, and to the animal's head at nasion, inion, and at the mastoid processes. After registering to a standard rhesus brain (Saleem, 2006), the images were used to guide VPL electrode implantations as described below. 
The detailed procedure for head-post and Blackrock electrode array (Blackrock Microsystems) implantation has been described previously (Chhatbar et al., 2010). In short, monkeys were anesthetized by using Ketamine $(20 \mathrm{mg} / \mathrm{kg})$, followed by intubation and respiration with isoflurane gas (1 - 3\%) and oxygen. After midline incision was made and the skin was retracted, two approximately $20 \mathrm{~mm}$ by $20 \mathrm{~mm}$ bone flaps were removed on each side of the midline over the $\mathrm{S} 1$ hand region. After dura flaps were opened, S1 receptive fields were mapped with sharp electrodes (Microprobes Com.) by lightly touching and manipulating finger pads, palms, arms and shoulders. Then two platinum-iridum microelectrode arrays (10 by 10; electrode pitch 400 um and electrode length $1.5 \mathrm{~mm}$; Blackrock Microsystems) were pneumatically inserted in the 'hot spot' of the hand area that demonstrated clear receptive fields during the mapping process bilaterally for monkey A. One array was implanted in the left S1 (contralateral to the testing right hand) in monkey G. It was inserted close to the central sulcus on the postcentral gyrus (Fig1 B), thus placing it in area 1 and possibly some of area 2 . Then under the guidance of the above imaging and stereotaxic coordination (Paxinos et al., 2000), either a customized sharp electrode array ( 2 by 4 ; tip distance 500 um, in monkey G) or a 24 channel linear array (space 200 um; LMA Microprobes, in monkey A) was lowered straight from the cortex to the VPL (Fig 1C). Similar to S1 mapping, receptive fields in left VPL were also mapped and the VPL array was implanted in the 'hot spot' hand representation area. VPL and S1 neural responses showed overlapping receptive fields. In addition to the above electrode implantation, monkey A was further implanted with PVC guides for near-infrared spectroscopy (NIRS) recording in the frontal portion over the skull. The PVC guides were secured to implanted bone screws with translucent acrylic, and a top layer of acrylic was applied to seal the implant to the surrounding skin margins. Once the arrays were in the desired depth, bone cement mixed with antibiotic Gentamicin (Palacos R, Zimmer Inc., Warsaw, IN, USA) was built around the connectors of the array, and the exposed skull was closed. For monkey G, all the electrode connectors were anchored to the 
skull with cement instead of our customary platform. At the end of the surgery, the surgical area was cleaned and topical triple antibiotic gel was applied to the surgical wound.

Following the surgery, analgesics (Rimadyl, $2 \mathrm{mg} / \mathrm{Kg}$; Buprenex, $0.02 \mathrm{mg} / \mathrm{Kg}$ ) and antibiotics (Baytril, 6mg/Kg) were administered for 3 and 5 days, respectively. Bicillin (50000 i.u./kg) was administered every other day for 14 days. Recordings began three weeks after implantation. In this paper, we present data only from the recordings of the left S1 and left VPL, both of which are contralateral to the stimulated right hand. At the end of the final recording session, strong electrolytic lesions were made in monkey $(G)$ who was sacrificed by first anesthetizing with ketamine (5-20 mg/kg, IM) and subsequently sodium pentobarbital (I.V., $100 \mathrm{mg} / \mathrm{Kg}$ ) injection. Following the complete loss of corneal and cutaneous reflexes the thorax was opened and the left ventricle was catheterized for introduction of saline into the arterial vasculature. The right atrium was cut to allow blood and then saline to flow out of the body. Subsequently, the animal was perfused through the heart, first with $0.9 \%$ saline, followed by $3.5 \%$ paraformaldehyde in $0.1 \mathrm{M}$ phosphate buffer $(\mathrm{pH}=7.4)$. The brain was removed from the skull and kept overnight in the same fixative solution. It was then immersed in $20 \%$ sucrose in $0.1 \mathrm{M}$ phosphate buffer $(\mathrm{pH}=7.4)$. Subsequently, serial $40-80 \mathrm{~mm}$-thick brain sections were cut using either a vibratome or a freezing microtome, and electrode placements were confirmed. As monkey A is still being utilized for research, it has no histological confirmation at this moment.

\subsection{Neural recording and offline sorting}

In each recording session, monkeys were seated comfortably in a non-human primate chair within a soundproof chamber. Neural data were acquired through a unity gain head-stage (Plexon Inc.) with a multichannel acquisition processor (Plexon Inc.). Neural signals were bandpass filtered $(100-8.8 \mathrm{KHz})$ and digitized at a sampling frequency of $40 \mathrm{kHz}$. Spikes were detected online when above a threshold (3 to 4 S.D) baseline noise level. Online detected spike waveforms were further classified offline as single units (SUs) and multi-units (MUs) using a 
commercial Offline Sorter (Plexon Com.). SUs were defined subjectively by the presence of clear waveform shapes with interspike intervals $>1.6 \mathrm{~ms}$, and good separation in principal components feature space. Typical waveforms and interspike intervals (ISIs) of isolated SUs in S1 and VPL are shown in figure $1 \mathrm{C}$ and D, respectively. Units that do not show clear isolation were taken as multi-units (MUs). Similar to our previous report (Song and Francis, 2013), we found that SU and the combined (SU/MU) data showed similar patterns, however to avoid the possible bias of synchrony coding (described below) to MUs, we only used well isolated SUs in our analysis. Up to 46 SUs on the S1 array and 9 SUs on the VPL array were isolated in each session.

Mechanical and electrical stimulators: Tactile stimulus was generated as an indent around 1 $\mathrm{mm}$ depth in the finger pad for $0.3 \mathrm{~s}$ using a computer-controlled solenoid actuator $(1 \mathrm{~mm}$ diameter) (Fig 1 A). Electrical stimulus was delivered via an isostimulator (Model 2200, A-M Systems Inc.) to a pair of stimulating electrodes in VPL. Both the tactile and electrical stimulators were controlled by a personal computer via a digital card ( $\mathrm{PCl}-6229$, National Instruments Inc.) running a customized software (Mathworks, Inc.). The electrical stimulator was capable of generating different patterns of stimuli (such as pulse width, amplitude and frequency). It was also able to stimulate in a closed-loop configuration, where stimulation was triggered by the firing of an online sorted cell (spike-triggered stimulation, in real-time), or from a pre-recorded spiking time series (random stimulation). The real-time control program for the digital card was written in C language (LabWindows / CVI, National Instruments Inc.).

\subsection{Testing protocols}

Before implantation surgeries, monkeys were well trained to be seated quietly in a primate chair with their right arms restrained to an exoskeletal robotic system (KINARM). In each session, no behavioral task was involved, and we chose the finger pads from which clear neural responses were observed (monitored from sound and oscilloscope) in both VPL and S1. Two protocols 
were tested, as displayed in Figure $1 \mathrm{~F}$, Protocol 1 was used to investigate tactile encoding of unit activities in S1 and VPL. In this protocol, 180 tactile stimuli were randomly delivered to a finger pad at a rate from $0.5 \mathrm{~Hz}$ to $2 \mathrm{~Hz}$ in each session. Unit activities in S1 and VPL were recorded simultaneously, and tactile information encoded in either firing rate or synchrony was calculated. Protocol 2 was used to study the plasticity of thalamocortical circuits after 30 minutes of neuropresthetic electrical stimulation in VPL. The delivery of electrical stimulation pulses in VPL was triggered either by online sorted spikes from a reference unit in S1 (closedloop/spike-triggered stimulation) or an offline pre-recorded spiking time series of a S1 unit (random stimulation). This "random stimulation" kept the same type of statistical stimulation pattern as the closed-loop paradigm, but decoupled in time from ongoing neural dynamics. That is the underlying state of the nervous system. In closed-loop stimulation, considering the latency from S1 to VPL (Shin and Chapin, 1990), electrical stimulus was delivered 5 ms after every S1 spike. To more likely induce neural responses from all triggering event, each stimulus was a cathode-leading biphasic double-pulse (amplitude: $50 \mu \mathrm{A}$; pulse width: $200 \mu \mathrm{s}$ per phase; pulse interval: $2 \mathrm{~ms}$ ). To avoid multiple stimulations from a single triggering events, only one stimulus was delivered within $5 \mathrm{~ms}$ of a triggering event. For possible use in somatosensory prosthetics applications, we applied this electrical stimulation hoping to induce high frequency doublets which are often seen in S1 in response to natural touch (Baker et al., 2003). To reduce current spread, bipolar VPL stimulation leads were chosen from two adjacent channels. There were about 18000 stimuli delivered in each 30 min session for a referencing unit with a mean firing rate of $10 \mathrm{~Hz}$. All triggering (S1) and stimulating (VPL) sites were randomly chosen from electrodes which had shown neural response during tactile stimulation. Unfortunately our recording setup did not allow us to record during electrical stimulation. Thus, we could not ascertain whether or not VPL electrical stimulation evoked responses in the triggering S1 cell. No cutaneous stimuli were delivered during either STS or random stimulation, and the monkeys were seated quietly during both periods. To test the plasticity of the thalamocortical circuit and 
thereby find a suitable stimulation protocol for neuroprosthetic applications, neural responses were tested by tactile stimulation of the same finger pad both immediately before and after VPL electrical stimulation (as in protocol 1). In each session, there was an interval of at least 30 minutes between closed-loop and random VPL stimulation. Based on our previous observations, this would bring the network to pre-stimulation level (Song et al., 2011). The information encoded and transferred in VPL and S1 with the two encoding strategies and different stimulation conditions were subsequently compared.

\subsection{Data Analyses}

\subsubsection{Tactile information encoded in firing rate and synchrony}

To quantitatively evaluate the efficiency of sensory encoding, two putative neural encoding strategies were tested in individual unit (rate coding) and unit pairs (synchrony coding) within the framework of information theory. Synchrony was defined as simultaneous firing of two units within $1 \mathrm{~ms}$ timescale bin. Mutual-information between neuronal responses (rate or synchrony) and stimuli is defined as

$$
I(\boldsymbol{R}, s)=\sum_{\boldsymbol{R}} \sum_{s} p(\boldsymbol{R}, s) \log _{2}\left(\frac{p(\boldsymbol{R}, s)}{p(\boldsymbol{R}) \cdot p(s)}\right)
$$

where $\boldsymbol{R}$ represents neural response matrix to tactile stimulus. It is either the spike count of an individual unit or the number of synchronous events between unit pairs within the response window; $s$ is tactile stimulus, a binary state (touch or no-touch) in our experiment. $p(\boldsymbol{R})$ and $p(s)=0.5$ are the marginal probability distribution functions of $\boldsymbol{R}$ and $s$, respectively; $p(\boldsymbol{R}, s)$ is the joint probability distribution function of $\boldsymbol{R}$ and $s$. Similar to our previous report (Song and Francis, 2013), a 50 ms window after the stimulus onset showed optimal mutual-information. Thus the mutual-information between tactile stimuli and the firing of individual units or synchrony of unit pairs was calculated within this window. Information calculated with the empirical probability distribution can have bias when the number of trials is 
limited. As there were 180 responses (rate or synchrony coding) for each stimulus (touch vs non-touch), the bias was estimated and reliably corrected with Panzeri-Treves method (Panzeri and Treves, 1996). We further used a bootstrap method to test the significance; the mean of the bootstrapped mutual-information was further subtracted (Magri et al., 2009).

\subsubsection{Information transfer}

In order to characterize the connection between two neurons, linear cross-correlation is commonly used. However, transfer entropy (TE), which takes into account linear and nonlinear components, provides a general way to define causality strength between two spike trains. It has been applied to investigate information transmission between different cortical areas (Gourevitch and Eggermont, 2007). The TE from unit $X_{1}$ (time series $X_{1}^{n}$ ) to unit $X_{2}$ (time series $\left.X_{2}^{n}\right)$ is defined as

$$
T E_{X 1 \rightarrow X 2}=I\left(X_{2}^{n+1}, X_{1}^{n-1} \mid X_{2}^{n-1}\right)
$$

where $I$ is the mutual-information between $X_{1}^{n-1}$ and $X_{2}^{n+1}$ when $X_{2}^{n-1}$ is known. $X_{i}^{n}$ indicates non-overlapping binned spike count time series of unit $i$ at current time $n, X_{i}^{n-1}$ for a previous time and $X_{i}^{n+1}$ for the next time step. Accordingly, the TE between each pair of VPL and S1 units was calculated by binning their spike trains with different windows. Ideally the window length for both the causing and the caused signal should be optimized independently for each unit pair (Gourevitch and Eggermont, 2007). However, considering the large amount of possible unit pairs (up to 1500 for a single session), we chose the same window length for both the causing and the caused signal. The optimal TE window $(100 \mathrm{~ms})$, which showed maximum information for both causing and caused units (Fig 6C), was used in the following information flow calculation (see Fig $6 \mathrm{C}$ ). Following the procedure by Gourevitch (Gourevitch and Eggermont, 2007), the TE

$$
N T E_{X 1 \rightarrow X 2}=\left(T E_{X 1 \rightarrow X 2}-T E_{X 1 \rightarrow X 2}^{\text {shuffled }}\right) / H\left(X_{2}^{n+1} \mid X_{2}^{n-1}\right)
$$


$H\left(X_{2}^{n+1} \mid X_{2}^{n-1}\right)$ is the entropy of the process $X_{2}^{n+1}$ conditioned on its past.

Then the direction of information flow (DIF) from $X_{1}$ to $X_{2}$ was calculated as

$$
D I F_{X 1 \rightarrow X 2}=\left(N T E_{X 1 \rightarrow X 2}-N T E_{X 2 \rightarrow X 1}\right) /\left(N T E_{X 1 \rightarrow X 2}+N T E_{X 2 \rightarrow X 1}\right)
$$

Similar to the correlation coefficient, the value of $D I F_{X 1 \rightarrow X 2}$, which is within $(-1,+1)$, represents a statistical causality strength from $X_{1}$ to $X_{2}:+1$ indicates unidirectional flow from $X_{1}$ to $X_{2}$, while -1 signifies the reverse direction.

\subsubsection{Statistical analysis}

For individual unit analysis, similar patterns were found for different animals. Thus, we pooled all the data from the two monkeys together. The following statistics were tested: (1) parametric paired or unpaired two sample test (ttest or ttest2, Matlab) was used between different conditions for a normal distribution (tested with Kolmogorov-Smirnov), and rank test was employed otherwise. (2) two-sample Kolmogorov-Smirnov test (kstest2, MATLAB) was performed to compare whether two variables likely come from the same distribution or not. All the significance levels of the above tests were set at 0.05 , unless stated otherwise. All data analyses were performed using MATLAB (Math Works Inc.).

\section{Acknowledgements}

We thank Dr. Joseph T. Francis for reading of the early draft and all his support, Pratik Chhatbar for surgical assistance, and DLAR for their excellent animal care. We would like to thank Dr. Rodolfo Ricart and the staff of the Research Animal Resource Center at Weill Cornell Medical College for their assistance with the MRI scans. Supported by DARPA contract \# N66001-10-C-2008.

\section{REFERENCES}


Adrian ED (1926) The impulses produced by sensory nerve endings: Part I. The Journal of physiology 61:49-72.

Alloway KD, Zhang M, Dick SH, Roy SA (2002) Pervasive synchronization of local neural networks in the secondary somatosensory cortex of cats during focal cutaneous stimulation. Exp Brain Res 147:227-242.

Baker SN, Curio G, Lemon RN (2003) EEG oscillations at $600 \mathrm{~Hz}$ are macroscopic markers for cortical spike bursts. J Physiol 550:529-534.

Bernander O, Koch C, Douglas RJ (1994) Amplification and linearization of distal synaptic input to cortical pyramidal cells. J Neurophysiol 72:2743-2753.

Bi GQ, Poo MM (1998) Synaptic modifications in cultured hippocampal neurons: dependence on spike timing, synaptic strength, and postsynaptic cell type. The Journal of neuroscience : the official journal of the Society for Neuroscience 18:10464-10472.

Blundon JA, Bayazitov IT, Zakharenko SS (2011) Presynaptic gating of postsynaptically expressed plasticity at mature thalamocortical synapses. J Neurosci 31:16012-16025.

Borst A, Theunissen FE (1999) Information theory and neural coding. Nature neuroscience 2:947-957.

Bruno RM, Sakmann B (2006) Cortex is driven by weak but synchronously active thalamocortical synapses. Science 312:1622-1627.

Buonomano DV, Merzenich MM (1998) Cortical plasticity: from synapses to maps. Annual review of neuroscience 21:149-186.

Camarillo L, Luna R, Nacher V, Romo R (2012) Coding perceptual discrimination in the somatosensory thalamus. Proc Natl Acad Sci U S A 109:21093-21098.

Castro-Alamancos MA (2002) Properties of primary sensory (lemniscal) synapses in the ventrobasal thalamus and the relay of high-frequency sensory inputs. J Neurophysiol 87:946-953.

Chhatbar PY, von Kraus LM, Semework M, Francis JT (2010) A bio-friendly and economical technique for chronic implantation of multiple microelectrode arrays. Journal of neuroscience methods 188:187-194.

Chun S, Bayazitov IT, Blundon JA, Zakharenko SS (2013) Thalamocortical long-term potentiation becomes gated after the early critical period in the auditory cortex. J Neurosci 33:7345-7357.

Constantinople CM, Bruno RM (2013) Deep cortical layers are activated directly by thalamus. Science 340:1591-1594.

da Costa NM, Martin KA (2009) The proportion of synapses formed by the axons of the lateral geniculate nucleus in layer 4 of area 17 of the cat. J Comp Neurol 516:264-276.

Dan Y, Poo MM (2004) Spike timing-dependent plasticity of neural circuits. Neuron 44:23-30.

Datwani A, Iwasato T, Itohara S, Erzurumlu RS (2002) Lesion-induced thalamocortical axonal plasticity in the S1 cortex is independent of NMDA receptor function in excitatory cortical neurons. J Neurosci 22:9171-9175.

Diamond ME, Huang W, Ebner FF (1994) Laminar comparison of somatosensory cortical plasticity. Science 265:1885-1888.

Douglas RJ, Koch C, Mahowald M, Martin KA, Suarez HH (1995) Recurrent excitation in neocortical circuits. Science 269:981-985.

Feldman DE (2009) Synaptic mechanisms for plasticity in neocortex. Annu Rev Neurosci 32:33-55.

Feldman DE, Nicoll RA, Malenka RC (1999) Synaptic plasticity at thalamocortical synapses in developing rat somatosensory cortex: LTP, LTD, and silent synapses. J Neurobiol 41:92-101.

Fox K, Glazewski S, Schulze S (2000) Plasticity and stability of somatosensory maps in thalamus and cortex. Curr Opin Neurobiol 10:494-497.

Ganguly K, Carmena JM (2009) Emergence of a stable cortical map for neuroprosthetic control. PLoS biology 7:e1000153. 
Ganguly K, Poo MM (2013) Activity-dependent neural plasticity from bench to bedside. Neuron 80:729741.

Gautrais J, Thorpe S (1998) Rate coding versus temporal order coding: a theoretical approach. Biosystems 48:57-65.

Ghosh S, Murray GM, Turman AB, Rowe MJ (1994) Corticothalamic influences on transmission of tactile information in the ventroposterolateral thalamus of the cat: effect of reversible inactivation of somatosensory cortical areas I and II. Exp Brain Res 100:276-286.

Gourevitch B, Eggermont JJ (2007) Evaluating information transfer between auditory cortical neurons. J Neurophysiol 97:2533-2543.

Heming EA, Choo R, Davies JN, Kiss ZH (2011) Designing a thalamic somatosensory neural prosthesis: consistency and persistence of percepts evoked by electrical stimulation. IEEE Trans Neural Syst Rehabil Eng 19:477-482.

Huxter J, Burgess N, O'Keefe J (2003) Independent rate and temporal coding in hippocampal pyramidal cells. Nature 425:828-832.

Ito HT, Schuman EM (2007) Frequency-dependent gating of synaptic transmission and plasticity by dopamine. Front Neural Circuits 1:1.

Jackson A, Mavoori J, Fetz EE (2006) Long-term motor cortex plasticity induced by an electronic neural implant. Nature 444:56-60.

Jones EG (1991) The anatomy of sensory relay functions in the thalamus. Prog Brain Res 87:29-52.

Jones EG (2001) The thalamic matrix and thalamocortical synchrony. Trends Neurosci 24:595-601.

Jones EG, Pons TP (1998) Thalamic and brainstem contributions to large-scale plasticity of primate somatosensory cortex. Science 282:1121-1125.

Joseph E. O'Doherty MAL, Peter J. Ifft, Katie Z. Zhuang, Solaiman Shokur, Hannes Bleuler \& Miguel A. L. Nicolelis (2011) Active tactile exploration using a brain-machine-brain interface. Nature 479:228-231.

Kaas JH (1991) Plasticity of sensory and motor maps in adult mammals. Annu Rev Neurosci 14:137-167.

Kaas JH (1999) Is most of neural plasticity in the thalamus cortical? Proc Natl Acad Sci U S A 96:76227623.

Kaas JH, Merzenich MM, Killackey HP (1983) The reorganization of somatosensory cortex following peripheral nerve damage in adult and developing mammals. Annual review of neuroscience 6:325-356.

Keller A, Arissian K, Asanuma H (1992a) Synaptic proliferation in the motor cortex of adult cats after long-term thalamic stimulation. J Neurophysiol 68:295-308.

Keller A, Arissian K, Asanuma H (1992b) Synaptic proliferation in the motor cortex of adult cats after long-term thalamic stimulation. Journal of neurophysiology 68:295-308.

Komura Y, Tamura R, Uwano T, Nishijo H, Kaga K, Ono T (2001) Retrospective and prospective coding for predicted reward in the sensory thalamus. Nature 412:546-549.

Li L, Ebner FF (2007) Cortical modulation of spatial and angular tuning maps in the rat thalamus. J Neurosci 27:167-179.

Liu XB, Honda CN, Jones EG (1995) Distribution of four types of synapse on physiologically identified relay neurons in the ventral posterior thalamic nucleus of the cat. J Comp Neurol 352:69-91.

Magri C, Whittingstall K, Singh V, Logothetis NK, Panzeri S (2009) A toolbox for the fast information analysis of multiple-site LFP, EEG and spike train recordings. BMC Neurosci 10:81.

Montemurro MA, Panzeri S, Maravall M, Alenda A, Bale MR, Brambilla M, Petersen RS (2007) Role of precise spike timing in coding of dynamic vibrissa stimuli in somatosensory thalamus. J Neurophysiol 98:1871-1882. 
Nicolelis MA, Ghazanfar AA, Stambaugh CR, Oliveira LM, Laubach M, Chapin JK, Nelson RJ, Kaas JH (1998) Simultaneous encoding of tactile information by three primate cortical areas. Nature neuroscience 1:621-630.

Niebur E, Hsiao SS, Johnson KO (2002) Synchrony: a neuronal mechanism for attentional selection? Curr Opin Neurobiol 12:190-194.

Nikolaev A, Leung KM, Odermatt B, Lagnado L (2013) Synaptic mechanisms of adaptation and sensitization in the retina. Nat Neurosci 16:934-941.

Nudo RJ, Milliken GW, Jenkins WM, Merzenich MM (1996) Use-dependent alterations of movement representations in primary motor cortex of adult squirrel monkeys. J Neurosci 16:785-807.

O'Doherty JE, Lebedev MA, Ifft PJ, Zhuang KZ, Shokur S, Bleuler H, Nicolelis MAL (2011) Active tactile exploration using a brain-machine-brain interface. Nature 479:228-U106.

Oberlaender M, Ramirez A, Bruno RM (2012) Sensory experience restructures thalamocortical axons during adulthood. Neuron 74:648-655.

Pais-Vieira M, Lebedev MA, Wiest MC, Nicolelis MA (2013) Simultaneous top-down modulation of the primary somatosensory cortex and thalamic nuclei during active tactile discrimination. J Neurosci 33:4076-4093.

Panzeri S, Treves A (1996) Analytical estimates of limited sampling biases in different information measures. Network-Comp Neural 7:87-107.

Panzeri S, Petersen RS, Schultz SR, Lebedev M, Diamond ME (2001) The role of spike timing in the coding of stimulus location in rat somatosensory cortex. Neuron 29:769-777.

Parker AJ, Newsome WT (1998) Sense and the single neuron: probing the physiology of perception. Annu Rev Neurosci 21:227-277.

Paxinos G, Huang XF, Toga AW (2000) The rhesus monkey brain in stereotaxic coordinates. San Diego, CA: Academic Press.

Rebesco JM, Stevenson IH, Kording KP, Solla SA, Miller LE (2010) Rewiring neural interactions by microstimulation. Front Syst Neurosci 4.

Reed JL, Qi HX, Kaas JH (2011) Spatiotemporal properties of neuron response suppression in owl monkey primary somatosensory cortex when stimuli are presented to both hands. The Journal of neuroscience : the official journal of the Society for Neuroscience 31:3589-3601.

Reed JL, Pouget P, Qi HX, Zhou Z, Bernard MR, Burish MJ, Haitas J, Bonds AB, Kaas JH (2008) Widespread spatial integration in primary somatosensory cortex. Proc Natl Acad Sci U S A 105:10233-10237.

Reinagel P, Reid RC (2000) Temporal coding of visual information in the thalamus. J Neurosci 20:53925400

Rioult-Pedotti MS, Donoghue JP, Dunaevsky A (2007) Plasticity of the synaptic modification range. J Neurophysiol 98:3688-3695.

Romo R, de Lafuente V (2013) Conversion of sensory signals into perceptual decisions. Prog Neurobiol 103:41-75.

Roy A, Steinmetz PN, Hsiao SS, Johnson KO, Niebur E (2007) Synchrony: a neural correlate of somatosensory attention. J Neurophysiol 98:1645-1661.

Saleem K (2006) Atlas of the rhesus monkey brain in stereotaxic coordinates : a combined mri and histology, 1st Edition. Boston, MA: Elsevier.

Salinas E, Sejnowski TJ (2001) Correlated neuronal activity and the flow of neural information. Nat Rev Neurosci 2:539-550.

Shannon CE (1948) A mathematical theory of communication. Bell System Technical Journal 27.

Shin HC, Chapin JK (1990) Mapping the effects of SI cortex stimulation on somatosensory relay neurons in the rat thalamus: direct responses and afferent modulation. Somatosens Mot Res 7:421-434.

Song S, Miller KD, Abbott LF (2000) Competitive Hebbian learning through spike-timing-dependent synaptic plasticity. Nat Neurosci 3:919-926. 
Song W, Giszter SF (2011) Adaptation to a cortex-controlled robot attached at the pelvis and engaged during locomotion in rats. The Journal of neuroscience : the official journal of the Society for Neuroscience 31:3110-3128.

Song W, Francis JT (2013) Tactile Information Processing in Primate Hand Somatosensory Cortex (S1) during Passive Arm Movement. J Neurophysiol.

Song W, Semework M, Francis JT (2011) Neural network reorganization after microstimulation in VPL and somatosensory cortex. In: Society for the Neural Control of Movement Puerto Rico.

Song W, Kerr CC, Lytton WW, Francis JT (2013) Cortical plasticity induced by spike-triggered microstimulation in primate somatosensory cortex. PLoS One 8:e57453.

Stanley GB, Jin J, Wang Y, Desbordes G, Wang Q, Black MJ, Alonso JM (2012) Visual orientation and directional selectivity through thalamic synchrony. J Neurosci 32:9073-9088.

Steinmetz PN, Roy A, Fitzgerald PJ, Hsiao SS, Johnson KO, Niebur E (2000) Attention modulates synchronized neuronal firing in primate somatosensory cortex. Nature 404:187-190.

Suminski AJ, Tkach DC, Fagg AH, Hatsopoulos NG (2010) Incorporating feedback from multiple sensory modalities enhances brain-machine interface control. J Neurosci 30:16777-16787.

Vazquez Y, Salinas E, Romo R (2013) Transformation of the neural code for tactile detection from thalamus to cortex. Proc Natl Acad Sci U S A 110:E2635-2644.

Vazquez Y, Zainos A, Alvarez M, Salinas E, Romo R (2012) Neural coding and perceptual detection in the primate somatosensory thalamus. Proc Natl Acad Sci U S A 109:15006-15011.

Viaene AN, Petrof I, Sherman SM (2011) Synaptic properties of thalamic input to layers $2 / 3$ and 4 of primary somatosensory and auditory cortices. J Neurophysiol 105:279-292.

Vue TY, Lee M, Tan YE, Werkhoven Z, Wang L, Nakagawa Y (2013) Thalamic control of neocortical area formation in mice. J Neurosci 33:8442-8453.

Wang Q, Webber RM, Stanley GB (2010) Thalamic synchrony and the adaptive gating of information flow to cortex. Nat Neurosci 13:1534-1541.

Wang X, Merzenich MM, Sameshima K, Jenkins WM (1995) Remodelling of hand representation in adult cortex determined by timing of tactile stimulation. Nature 378:71-75.

Weng HR, Lee JI, Lenz FA, Schwartz A, Vierck C, Rowland L, Dougherty PM (2000) Functional plasticity in primate somatosensory thalamus following chronic lesion of the ventral lateral spinal cord. Neuroscience 101:393-401.

\section{FIGURE LEGEND}

Figure 1. Experimental protocols. A), Monkeys were seated comfortably in a non-human primate chair with their right arm and hand restrained on a KINARM (BKIN Technologies). The fingers were held in place by a customized cast that allows tactile stimuli to be delivered reliably from a solenoid plunger. Each tactile stimulus (one stroke of solenoid) was applied for $0.3 \mathrm{~s}$ on the skin, which indents the skin on the finger pad (either digit 2, 3 or 4) by $1 \mathrm{~mm}$. Neuronal activities were recorded from electrode arrays in S1 (10 by 10, Blackrock Microsystems) and 
VPL (B) (customized 2 by 4 in monkey G; or 24 sites linear array in monkey A, Microprobes Com.) (C). Waveforms (upper) and interspike intervals (lower) of example single units in VPL (D) and S1 (E) are shown. F), we used two protocols to test tactile encoding (protocol 1) and plasticity of the thalamocortical circuit (protocol 2). In protocol 1, 180 stimuli were randomly delivered via a tactile stimulator (A) to a finger pad at a mean frequency of $0.5 \mathrm{~Hz}$. In protocol 2 , VPL was electrically stimulated either contingent on the firing of a single S1 unit (closedloop/spike-triggered stimulation) or based on a pre-recorded firing of the S1 unit (random stimulation) for $30 \mathrm{~min}$. There were resting intervals of at least 30 minutes between the two VPL stimulation paradigms. Protocol 1 was applied before and after each VPL stimulation (in the same finger pad).

Figure 2. Responses to tactile stimulation of example units. Units in both VPL (A) and S1 (C) showed firing rate modulation to tactile stimulation. Raster plots (top frame in panels A and C) and corresponding PSTHs (bottom frame in each panel) show neuronal responses to tactile stimulation. Black and red dots in the raster indicate spiking of two different units. Each row represents one trial (there are 180 trials in total). The black and red lines in each PSTH are from the units with the same color. Along with firing rate modulation in individual units, synchrony between unit pairs in either VPL (B) or S1 (D) was also responsive to tactile stimulation. Synchrony was defined as simultaneous firing of two units within 1 ms window. The two units used in $\mathbf{B}$ and $\mathbf{D}$ were the same two units in $\mathbf{A}$ and $\mathbf{C}$. Each dot in the synchrony raster plot (top frames in panels B and D) represents a synchrony of the two units within the same trial. Similar to the above PSTH, peri-stimulus synchrony histograms (PSSH) are drawn for the synchrony raster plots (black lines in bottom frames of panels of $\mathbf{B}$ and $\mathbf{D}$ ). The shaded area in $\mathbf{B}$ or $\mathbf{D}$ represents the $95 \%$ confidence interval of the trial shuffled PSSH of the two units. Time zero indicates the stimulation onset. 
Figure 3. Tactile information encoded with rate coding and synchrony coding. Tactile information encoded in the firing rate of individual units $(\mathbf{A})$ or in synchronic unit pairs $(\mathbf{B})$ is significantly larger ( ${ }^{*} p<0.01$, for kstest2 and ttest2) in VPL than that in $\mathrm{S} 1$. Rate coding is significantly higher ( ${ }^{*} p<0.01$, for kstest2 and ttest2) than synchrony coding in both VPL and S1. Apart from the difference in information value, the number of significantly tactile-encoded units also show differences (C and $\mathbf{D})$. There is a greater percentage of tactile-encoded units in VPL than in S1. A higher percentage of units encode with rate coding than with synchrony coding in both VPL and S1.

Figure 4. Effect of VPL stimulation on neural encoding. In VPL, tactile information encoded with rate coding did not significantly change after either closed-loop (spike-triggered) or random playback stimulation. The effect of random stimulation on the neural encoding was not significantly different from closed-loop stimulation for either rate coding (A) or synchrony coding (B). In S1, closed-loop stimulation showed significantly different effects from random stimulation for both rate coding (C) and synchrony coding (D). Bar plot in each panel represents the change in the percentage of units that significantly encoded tactile stimuli with rate coding ( $\mathbf{A}$ and $\mathbf{C})$ or synchrony coding (B and D). The number of units that employed rate coding in VPL decreased by around $7 \%$ after random stimulation compared to a $5 \%$ increase after closed-loop stimulation (A). Similarly, there was a $3 \%$ increase in S1 rate-coding after closed-loop stimulation compared to a $3 \%$ decrease after random stimulation (C). For synchrony encoding, the change in the number of units was no more than $2 \%$ in either VPL (B) or S1 (D). ( ${ }^{*} p<0.01$, for kstest2 and ranksum).

Figure 5. Changes in firing rate correlation between unit pairs. Firing rate correlation coefficient between two conditioning pairs (between recording $S 1$ unit and stimulating VPL unit) significantly increased after closed-loop stimulation compared with random stimulation. The 
changes were larger between conditioning pairs than between non-conditioning pairs $\left({ }^{*} p<0.05\right.$, for signrank test).

Figure 6. Changes of information transmission. Example transfer entropy (TE) maps between unit pairs show causality from VPL to S1 (one causing-unit from stimulating VPL and the other caused-unit from recording S1) (A) and from S1 to VPL (causing unit from recording S1 and caused unit from stimulating VPL) (B). Only unit pairs with significant causality are plotted, and the non-significant pairs are set to zero as background color (significance was tested with bootstrapping: $n=20$ ). The binning window to calculate TE was optimized by choosing the maximum information in the whole population, thus window length was chosen as $100 \mathrm{~ms}$ (C). The direction of information flow (DIF) from VPL to S1 (see method) shows that there were significantly different outcomes to spike-triggered and random stimulation. Spike-triggered stimulation tends to increase $(p>0.5$ at $\mathrm{DIF}=0)$ the causality from VPL to $S 1$, while random stimulation tends to decrease $(p<0.5$ at $D I F=0)$ the causality from VPL to $S 1$. (D). Similar to TE, we only used significant values to calculate the DIF. Error bars in (C) indicate standard errors (S.E.). Unit of the color scale bar in (A) and (B) is bit, and each element in the map represents a unit pair, with hot colors representing big values. Dotted horizontal line in (D) indicates $p=0.5$. $\left({ }^{*} p<0.05\right.$, for kstest2).

Figure 7. Stability of thalamocortical circuit. The sensory transmission gain $(K)$, which is ratio of the ensemble neural response from $\mathrm{S} 1$ to the ensemble neural response from VPL during tactile stimulation, was significantly ( $p<0.05$, regression) correlated between $K_{\text {pre }}$ and $K_{\text {post }}$ for both spike-triggered stimulation $(\mathbf{A})$ and random stimulation (B) when regressed $K_{\text {pre }}$ on $K_{\text {post }}$. Only spike-triggered stimulation demonstrates a regression line with a unit slope and zero interception, which indicates stability of the underlying circuit. Each point in the figure represents sensory transmission gain before (X-axis) and after ( $\mathrm{Y}$-axis) stimulation in each testing session, 
and the gray line indicates the regression line. $R^{2}$ indicates the R-squared value of the regression. 
A

Arm \& finger restraint

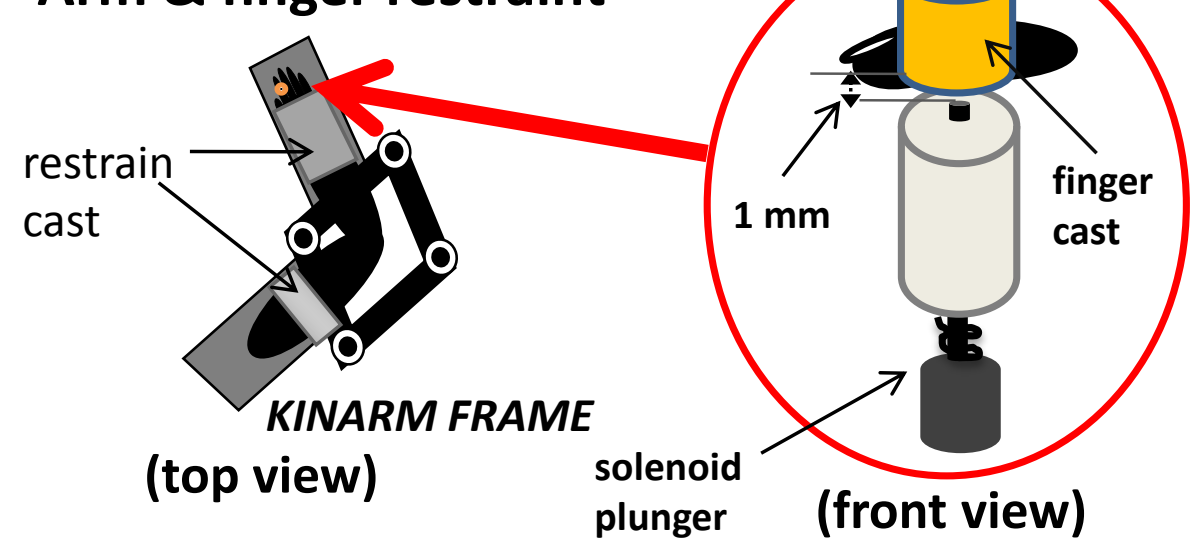

F

\section{Protocol 1}

$\prod_{\frac{3}{3}}^{0.3 \mathrm{~s}} \begin{gathered}3 \min \\ \text { - }\end{gathered}$

B
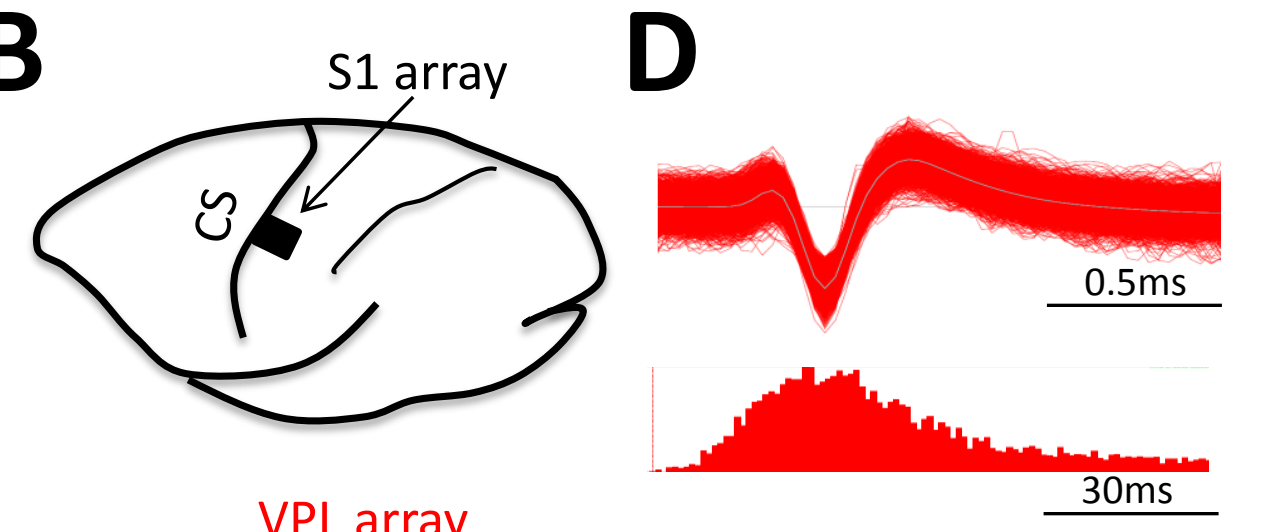

c
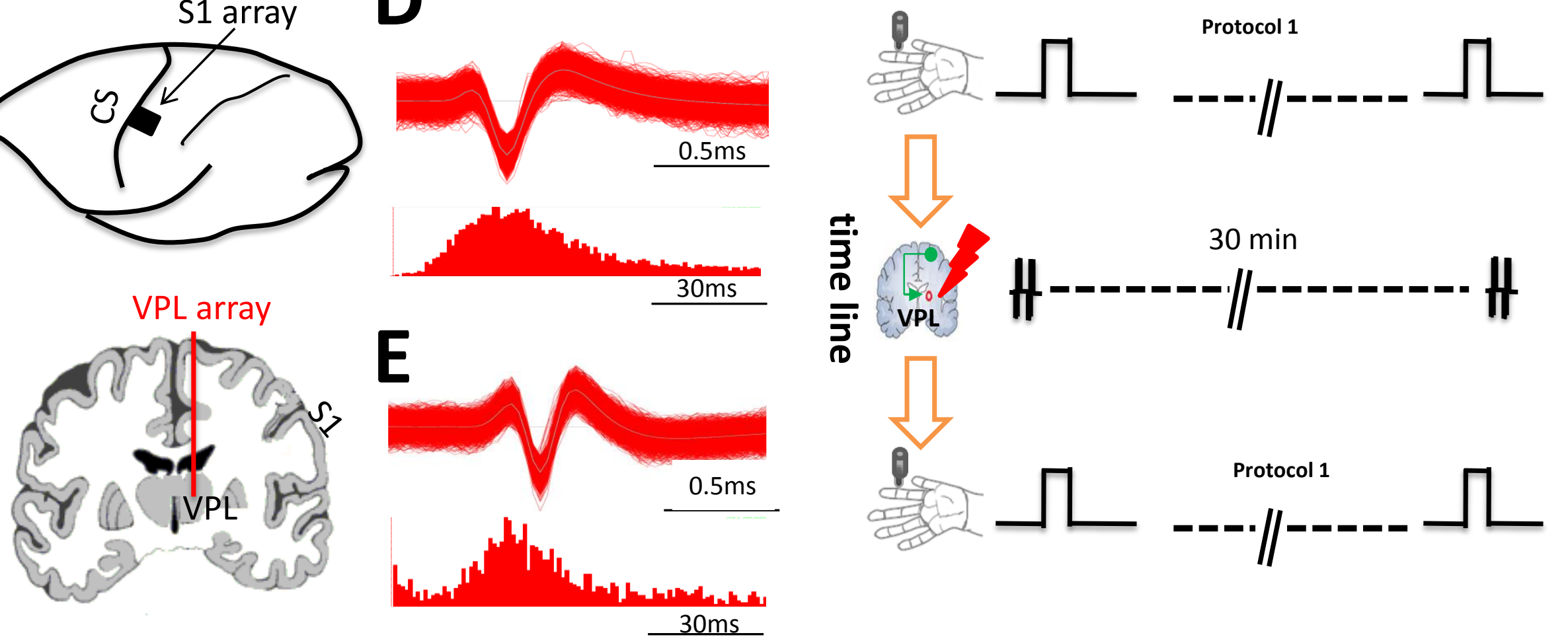
A

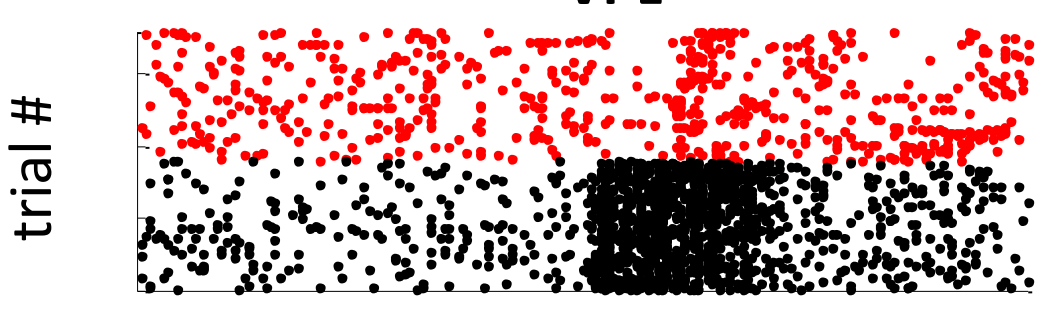

—unit A - unit B

200

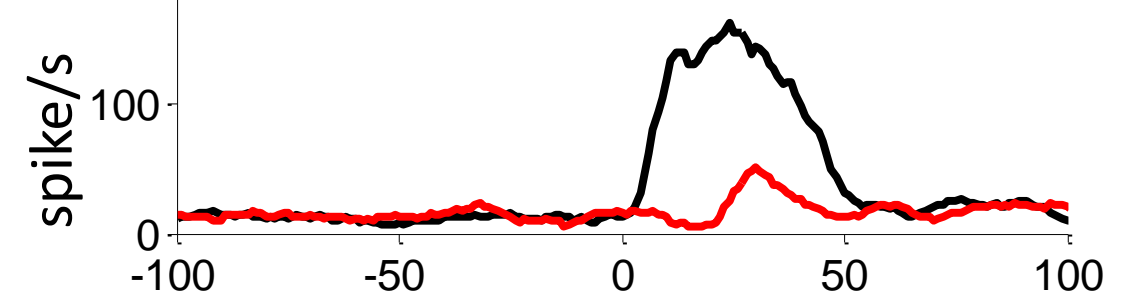

B
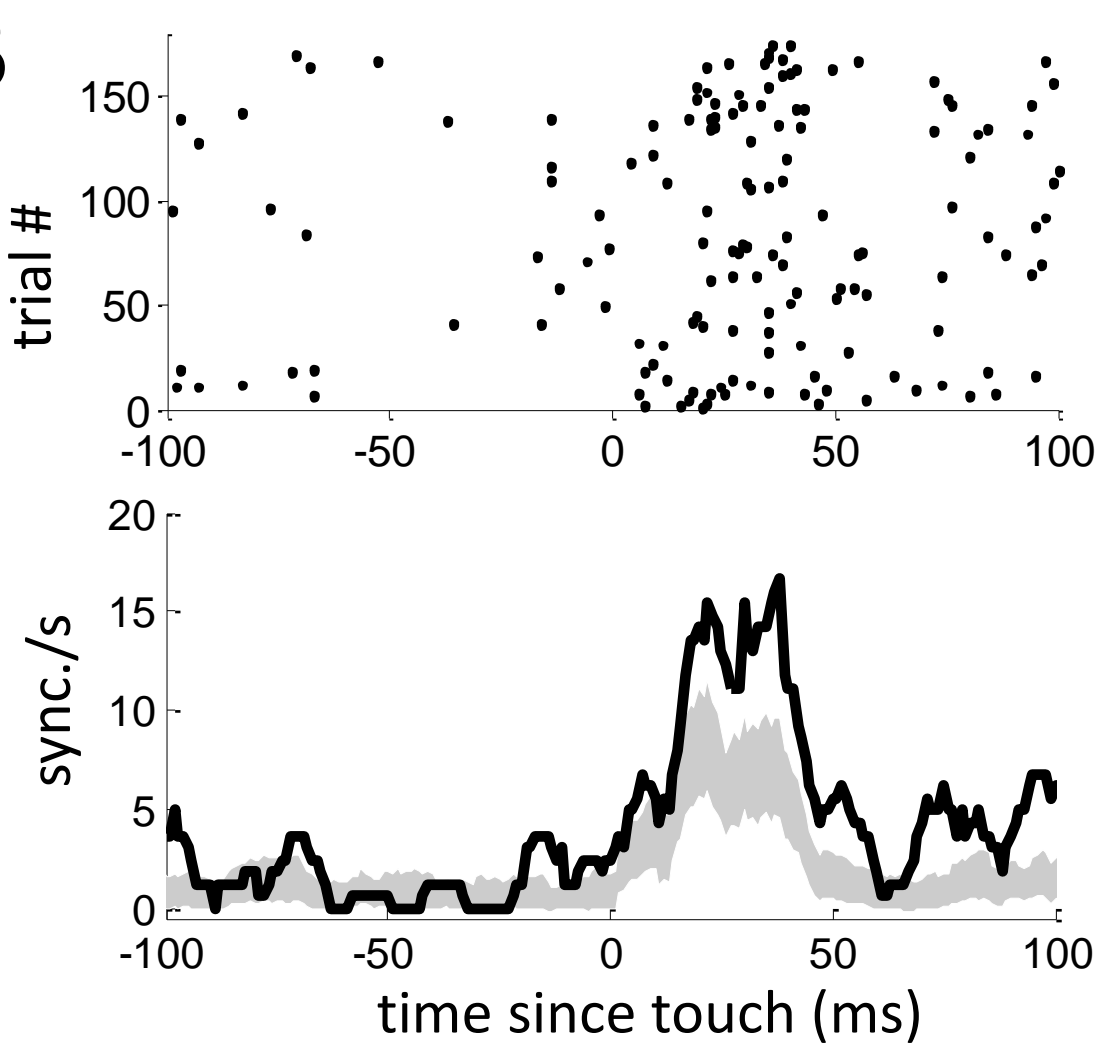

S1

Fig 2

$$
200
$$

$\longrightarrow$ unit $A=$ unit $B$

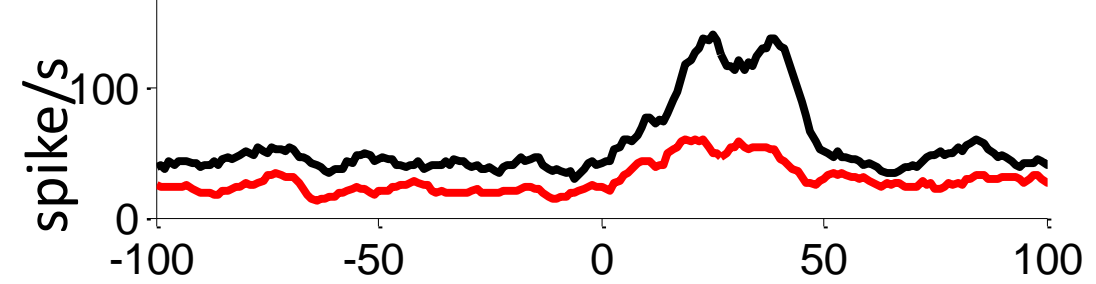

D

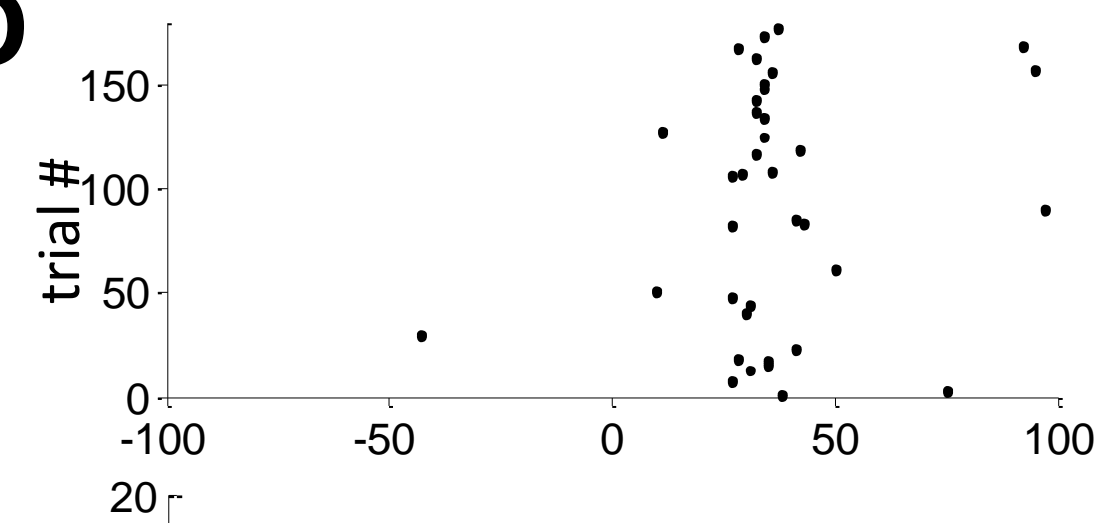

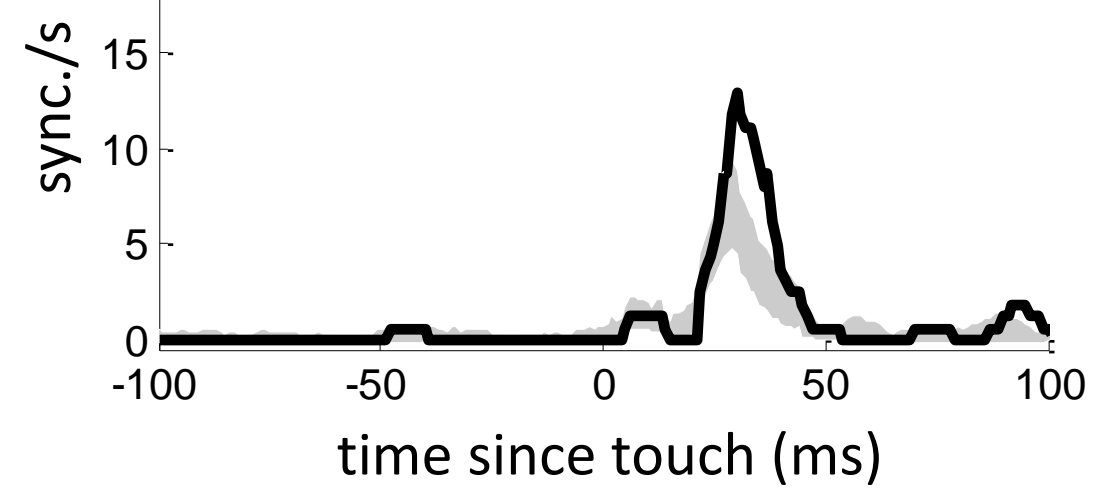


$\mathbf{A}$

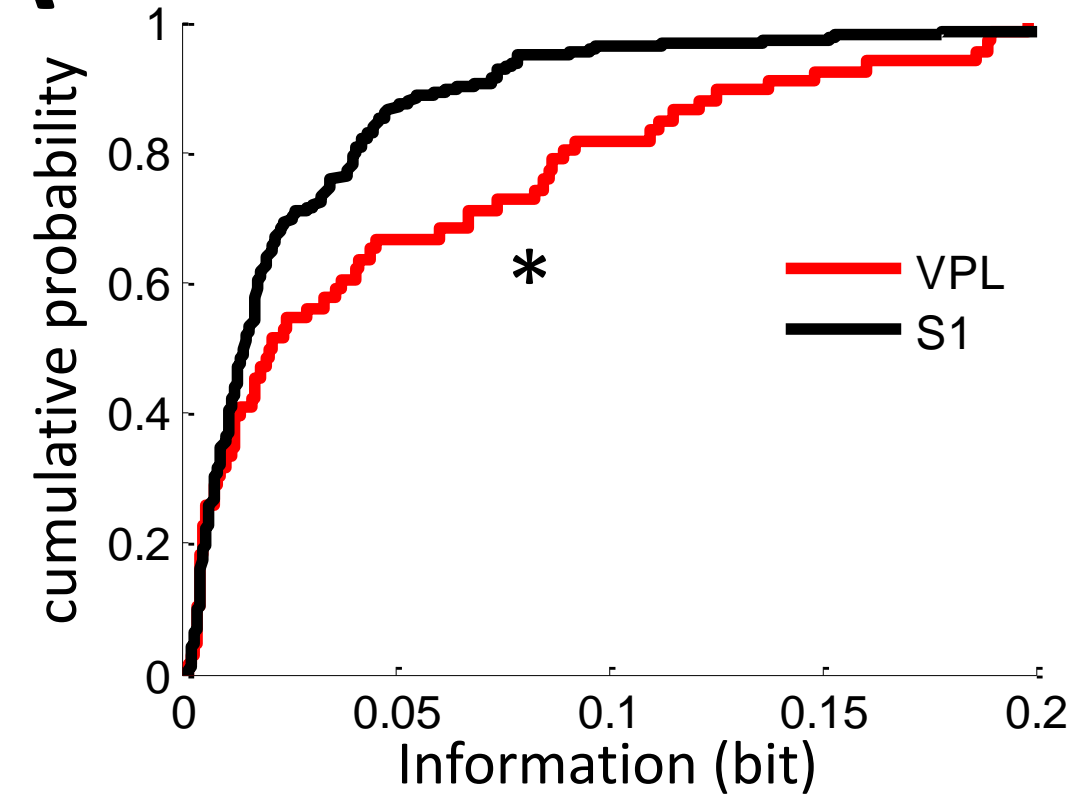

B

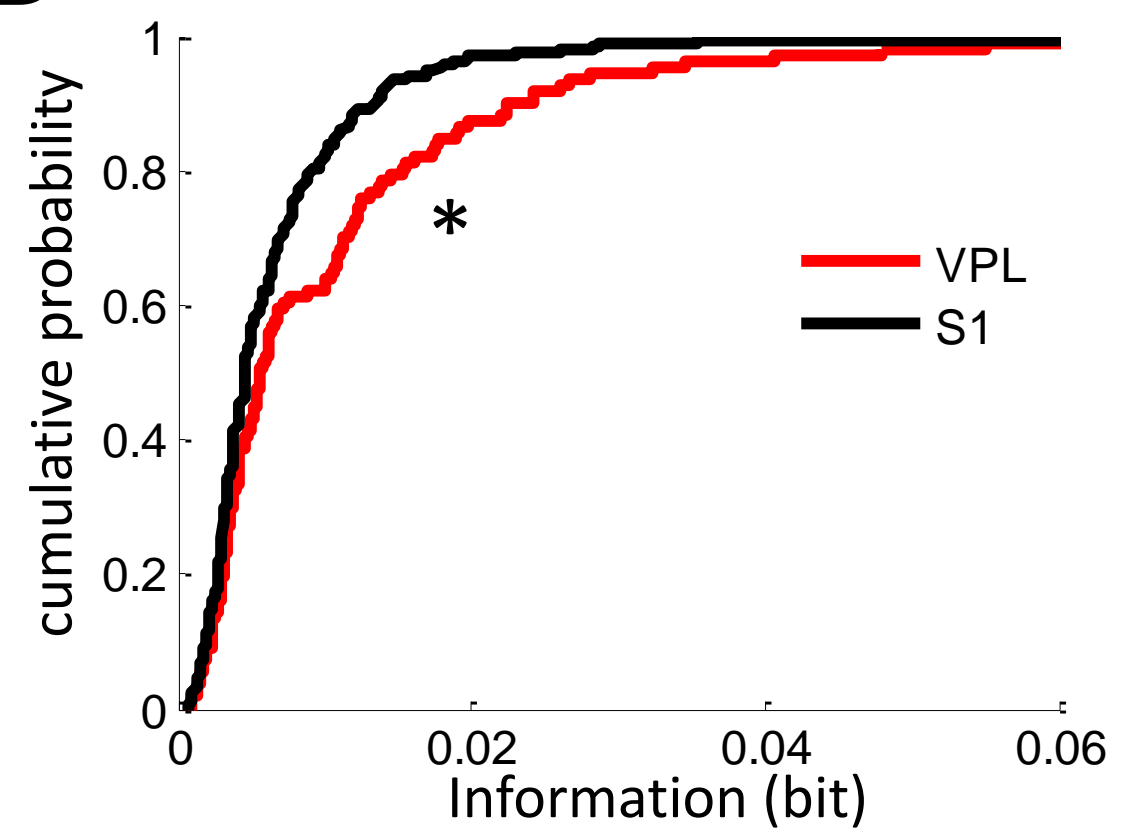

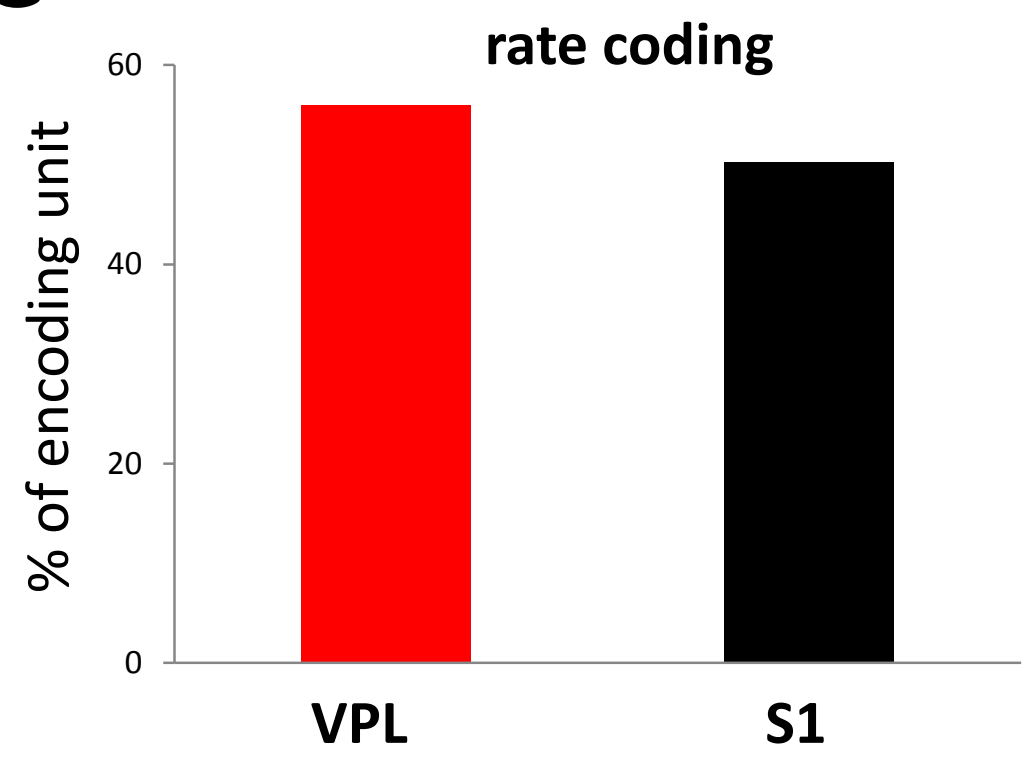

D

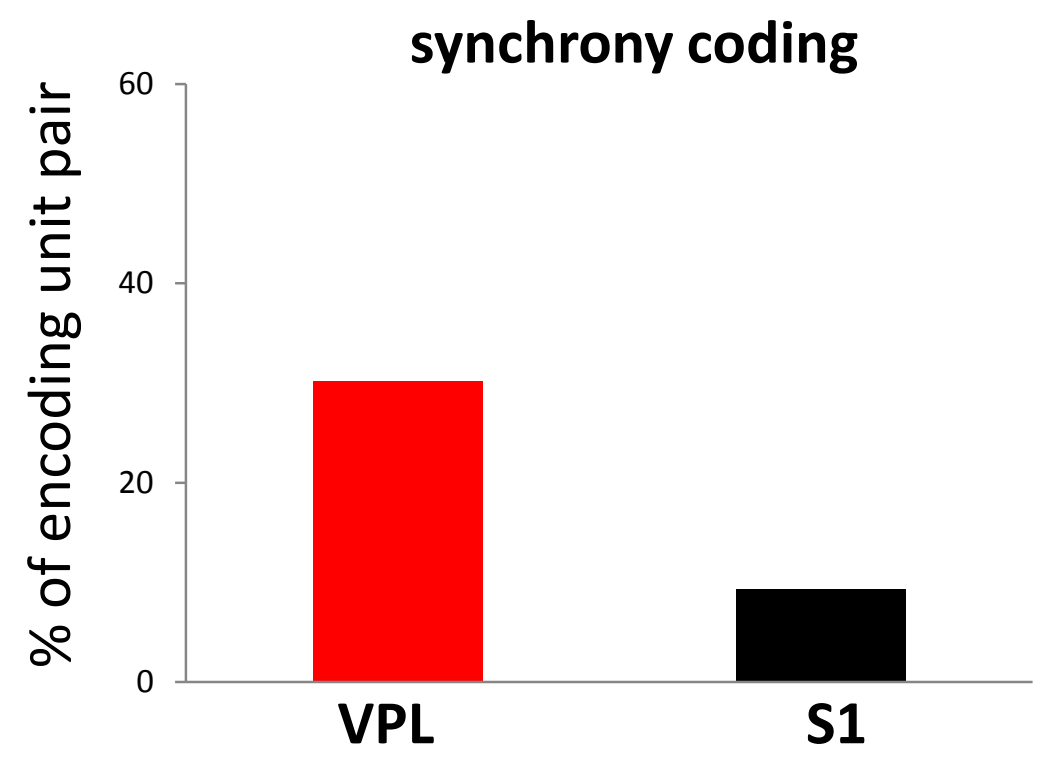


Fig 5

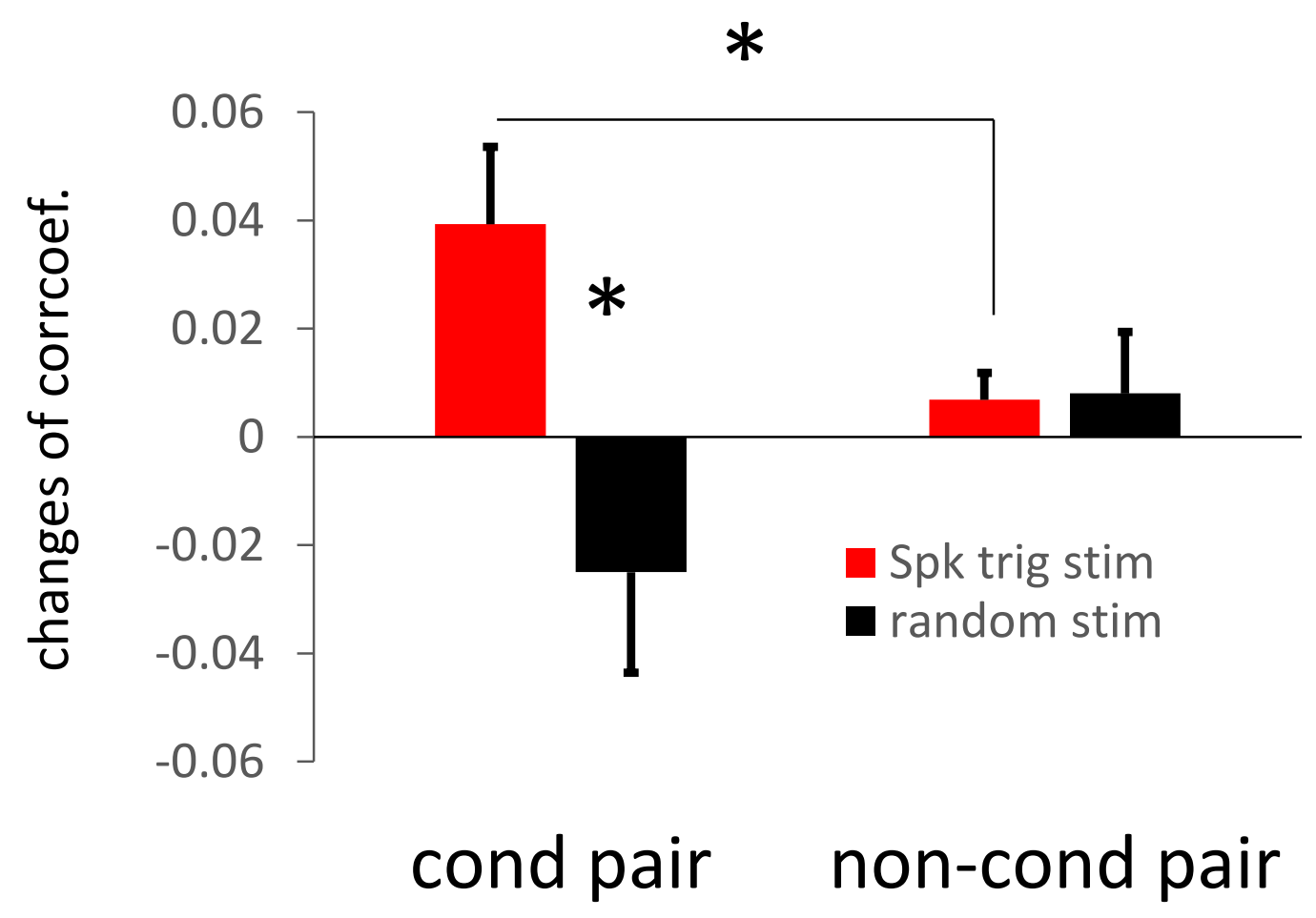



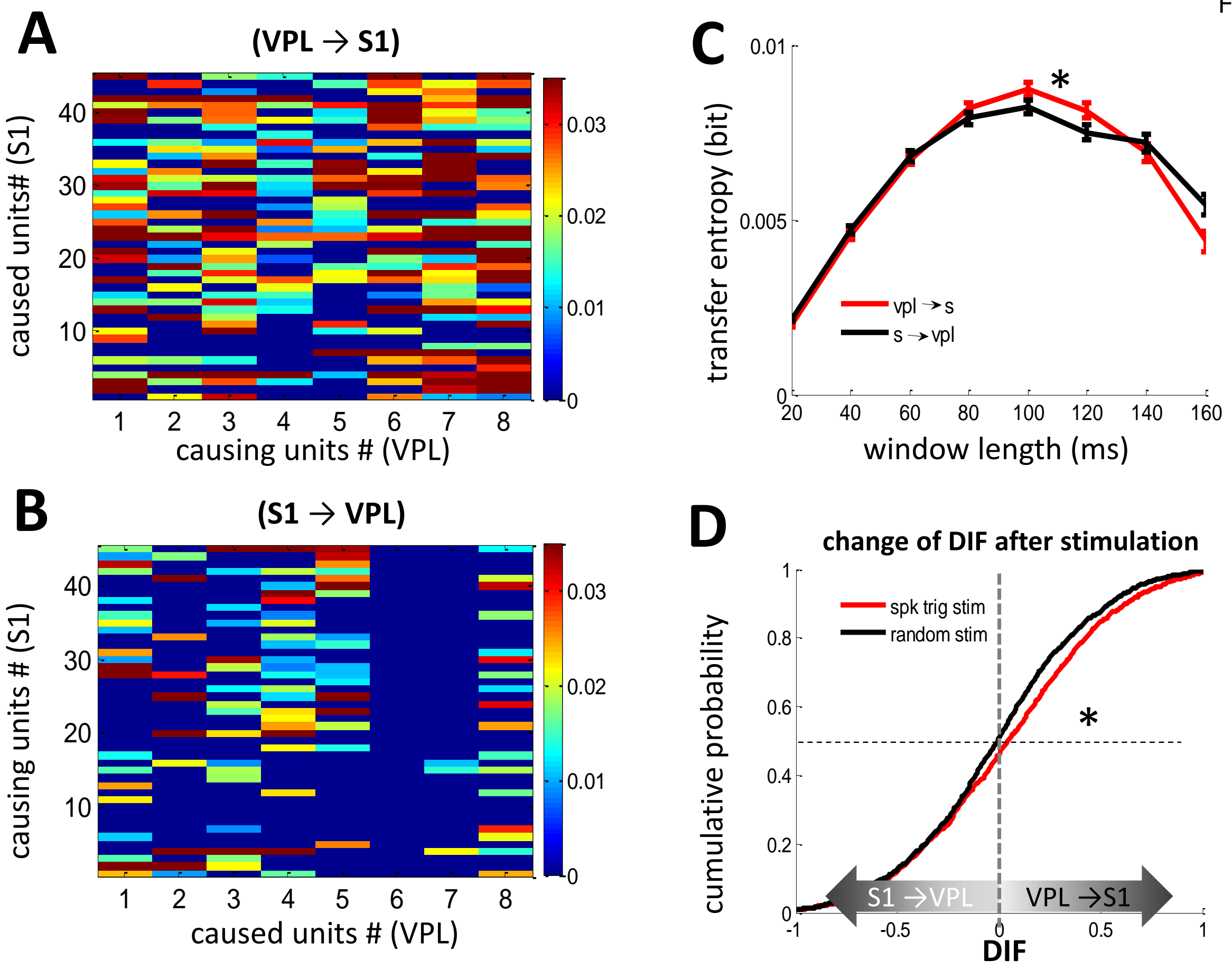

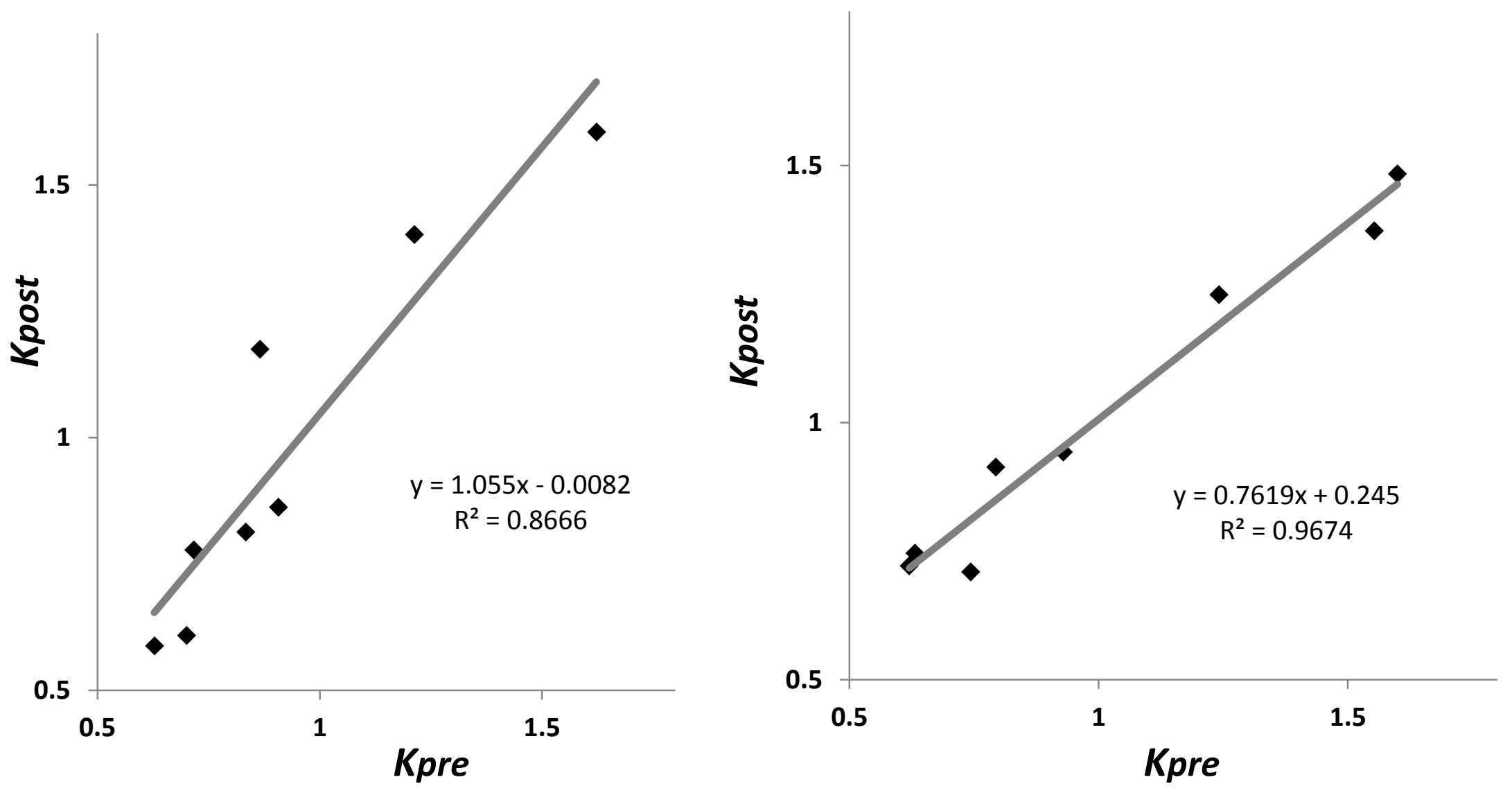